\title{
Article \\ An Alternative to Transvenous Lead Extraction in Selected Patients with CIED Infections-A Retrospective Outcome Study
}

\author{
Jennifer Llewellyn ${ }^{1, *(D)}$, Goutham Meda ${ }^{2}$, Daniel Garner ${ }^{1}$, David J. Wright ${ }^{1}$ and Archana Rao ${ }^{1}$ (D) \\ 1 Department of Cardiology, Liverpool Heart and Chest Hospital, Thomas Drive, Liverpool L14 3PE, UK; \\ danielrobert.garner@lhch.nhs.uk (D.G.); jay.wright@lhch.nhs.uk (D.J.W.); archana.rao@lhch.nhs.uk (A.R.) \\ 2 School of Medicine, University of Liverpool, Cedar House, Ashton Street, Liverpool L69 3GE, UK; \\ g.meda@student.liverpool.ac.uk \\ * Correspondence: jennifer.llewellyn@lhch.nhs.uk
}

check for updates

Citation: Llewellyn, J.; Meda, G.; Garner, D.; Wright, D.J.; Rao, A. An Alternative to Transvenous Lead Extraction in Selected Patients with CIED Infections-A Retrospective Outcome Study. Hearts 2022, 3, 6-13. https://doi.org/10.3390/

hearts3010002

Academic Editor:

Matthias Thielmann

Received: 21 December 2021

Accepted: 18 January 2022

Published: 20 January 2022

Publisher's Note: MDPI stays neutral with regard to jurisdictional claims in published maps and institutional affiliations.

Copyright: (c) 2022 by the authors. Licensee MDPI, Basel, Switzerland. This article is an open access article distributed under the terms and conditions of the Creative Commons Attribution (CC BY) license (https:// creativecommons.org/licenses/by/ $4.0 /)$.

\begin{abstract}
Cardiac implantable electronic device (CIED) implants are rising in an older, more comorbid population. The prevalence of CIED infection ranges from 1-4\%. Whilst complete extraction of all transvenous hardware is recommended for infected, eroded, or pre-eroding CIEDs, this approach is not without risk and may be unacceptable to some patients. Long-term data on a more conservative strategy is lacking. We report on our experience of conservative management with pocket revision as a primary strategy in carefully selected patients. Method: A retrospective review of all CIED revision procedures was undertaken at a large tertiary center, over a 7-year period, with a mean follow-up timeframe of 39 months. Results: A total of 86 patients underwent 96 revision procedures; 7 patients required further revisions and 13 went on to undergo CIED extraction by the end of the follow-up period. The overall rate of mortality at 12 months was $8.1 \%$, increasing to $24.4 \%$ at the end of the follow-up period. Conclusion: Our data provide important outcome information on an alternative strategy to lead extraction in carefully selected patients where the risk of extraction is perceived to be unacceptable. The absence of systemic infection appears to predict better outcomes than previously reported, and over two-thirds of patients remained complication-free at 12 months.
\end{abstract}

Keywords: cardiac implantable electronic devices; submuscular reburial; transvenous lead extraction; pacemakers; CIED infection

\section{Introduction}

Cardiac implantable electronic devices (CIEDs) are used to treat cardiac arrhythmias and heart failure [1]. The number of CIEDs implanted is increasing annually [2]. A total of $70 \%$ of CIED recipients are elderly (age 65 years or older), and over $75 \%$ have one or more coexisting medical conditions [3].

The reported prevalence of CIED infection in registries and national databases ranges from $1-4 \%$ [4-6].

As per the 2019 International CIED Infection Criteria, definite CIED clinical pocket infection involves pocket swelling, erythema, warmth, pain, purulent discharge/sinus formation or deformation of the pocket, adherence, and threatened erosion or an exposed generator or proximal leads $[7,8]$.

The recommended treatment for definite CIED infection involves the complete removal of all transvenous hardware $[1,7,9]$, but transvenous lead extraction (TLE) is a complex procedure that carries risks. Whilst the overall success rate of experienced hands is currently $>95 \%$ and major risks occur in $<2 \%$ of cases $[10,11]$, real-life outcomes may be less favorable, especially among frail elderly patients with multiple comorbidities.

Certain patient and lead factors may influence complications from extraction procedures. The number of leads extracted (each lead translating to a 3.5-fold increase in 
risk for any complication) [12] and the lead dwell time have both been found to increase extraction risk.

Frailty has not been investigated as a predictor of risk in TLE but is likely to play a part in clinical decision-making. Markers of frailty, such as age and co-morbidity, have been shown in a French registry to influence the decision-making process in choosing between extraction versus lead abandonment [13]. There is growing evidence that an estimation of frailty should be utilised in prognostication, as increased frailty is associated with poorer clinical outcomes [14], which can be seen in both cardiac surgery and heart failure hospitalizations [15]. The Clinical Frailty Scale is a judgement-based frailty tool; it was originally a 7-point scale but was later updated to 9-point scale to evaluate specific domains, including co-morbidity, function, and cognition, in the generation of a frailty score [16].

The General Medical Council (GMC) recently published guidance on decision-making and consent. It stated that decision-making is an ongoing process focused on the exchange of relevant information specific to the individual patient [17]. Furthermore, the information provided to patients prior to consenting should include various options for treating or managing the condition-including the option to take no action-and the nature of each option, as well as the potential benefits, risks of harm, uncertainties about success, and the likelihood of success for each option.

Informed consent for lead extraction is a complex process, and guidelines recommend that the alternatives to TLE be discussed with patients along with the risks of extraction. Quoting a standard risk can be difficult due to variable clinical presentation, patient characteristics, and lead factors.

Alternative strategies, including pocket revision, closed-loop irrigation systems, and partial removal, have been explored with variable success in selected patients where the risk-benefit ratio of extracting the leads is felt to be unfavorable or due to patient choice $[18,19]$.

We report on an alternative strategy for CIED revision in carefully selected patients following the switch to electronic patient records (EPR) in 2013 at our centre. This approach was only employed in patients who were unwilling to undergo TLE (patient choice) or where TLE was deemed too high-risk (due to a combination of patient and lead factors). Frailty is viewed as a reason to recommend a conservative approach but the authors acknowledge that other factors may have been considered at the time of decision-making, such as a plan for re-implantation or temporary pacing as well as the device indication and additional comorbidity.

\section{Materials and Methods}

The centre is responsible for all complex device implants and follow-up, as well as all CIED revisions and lead extractions for the region. As a high-volume extraction centre, we average 65 extractions $/ 125$ leads per year. The extractions are performed by 2 operators with $>12$ years of experience.

CIED revision was defined as the excision of the eroded or pre-eroded area and submuscular reburial with primary closure with an absorbable monofilament suture. Debridement of the wound, irrigation of the pocket, and the use of TYRX antibacterial envelopes, when available, were undertaken at the operator's discretion.

Patients underwent a clinical examination and blood tests, including tests for Creactive protein (CRP) and white cell count (WCC). Blood cultures and echocardiograms were undertaken where there was clinical suspicion of systemic involvement or the patient was pyrexial. A risk-benefit ratio was assessed and-where the former was felt to outweigh the latter-a discussion with the patient was undertaken, and informed written consent was obtained in all cases. Revision was mainly considered in the absence of systemic infection or vegetation. 
All procedures were undertaken in a dedicated cardiac pacing theater, and a single dose of intravenous antibiotics was deployed prior to incision, as per our standard policy. The pocket was swabbed at the time of the procedure.

Local research approval was obtained, and a retrospective EPR review of all CIED revision procedures over a 7-year period from 2013 to 2020 was undertaken. The patients were identified from procedure codes and cross-checked with the hard copy pacing theatre logbook. All patients identified as undergoing this procedure were included in the study.

Details of clinical presentation, patient demography, and the type, number, and dwell time of the leads were collected. Clinical presentation, antibiotic therapy, the duration of hospitalization, prior and subsequent procedures, and mortality data were also gathered. Mortality data were obtained via the NHS Demographic Batch Service (DBS), which links patient data to the NHS Spine (https://digital.nhs.uk/services/spine, accessed on 15 January 2021).

Patients were reviewed in 2 pre-defined subgroups based upon the indication for the revision-erosion or pre-erosion. Erosion was defined as the device or lead having broken through the skin and therefore being exposed to the surface. Pre-erosion was defined as the superficiality of the device or lead associated with the tethering/discoloration of surrounding skin. The former is a definite indication for lead extraction, whilst the latter is a relative indication.

The outcomes assessed include morbidity as defined by repeat procedures (revisions and/or extraction) and mortality. Data were analysed using Microsoft Excel ${ }^{\circledR}$.

\section{Results}

A total of 86 patients underwent 96 revision procedures over a 7-year period between March 2013 and 2020. Overall, 55.8\% of patients were male and the mean age was $72.9+/-15.1$ (median $=77.5$ years). The mean Clinical Frailty Scale (CFS) score was 4.3. The mean number of leads was 2.44, with a mean dwell time of 118 months. Overall, $56(65 \%)$ patients had undergone a procedure (i.e., a new implant $(n=6)$, an upgrade of functionality $(n=6)$, lead revision $(n=1)$, or generator replacement $(n=43))$ in the 12 months prior to requiring a revision. The mean follow-up period was 39 months (range $=5-90$ months). No patients were lost to follow-up. See Table 1 below.

Table 1. Patient and device demographics.

\begin{tabular}{|c|c|c|}
\hline & Erosion $(n=24)$ & Pre-Erosion $(n=62)$ \\
\hline \multicolumn{3}{|l|}{ Patient Characteristics } \\
\hline \multicolumn{3}{|l|}{ Age } \\
\hline - Mean +/-SD & $84.5+/-9.67$ & $67.59+/-14.09$ \\
\hline Male gender $n(\%)$ & $18(75 \%)$ & $32(51.6 \%)$ \\
\hline \multicolumn{3}{|l|}{ Clinical Frailty Score } \\
\hline - Mean +/-SD & $4.98+/-1.54$ & $3.98+/-1.25$ \\
\hline \multicolumn{3}{|l|}{ Indication for device $n(\%)$} \\
\hline - SN disease & $8(33.3 \%)$ & $11(17.8 \%)$ \\
\hline - AV block & $11(45.8 \%)$ & $11(17.8 \%)$ \\
\hline - Heart failure & $3(12.5 \%)$ & $23(37.1 \%)$ \\
\hline - Ablation & $1(4.2 \%)$ & $2(3.2 \%)$ \\
\hline - 1ry prevention & $0(0 \%)$ & $2(3.2 \%)$ \\
\hline - 2ry prevention & $0(0 \%)$ & $9(14.5 \%)$ \\
\hline - Unknown & $1(4.2 \%)$ & $4(6.5 \%)$ \\
\hline $\begin{array}{l}\text { Procedure in prior } 12 \text { months } \\
n(\%)\end{array}$ & $11(45.83 \%)$ & $44(70.97 \%)$ \\
\hline Prior sternotomy $n(\%)$ & $1(4.17 \%)$ & $4(6.45 \%)$ \\
\hline General anaesthetic $n / 96(\%)$ & $27 / 31(87.1 \%)$ & $55 / 63(87.3 \%)$ \\
\hline \multicolumn{3}{|l|}{ Length of stay (days) } \\
\hline Mean +/-SD & $3.19+/-4.12$ & $0.27+/-0.97$ \\
\hline
\end{tabular}


Table 1. Cont.

\begin{tabular}{lcc}
\hline & Erosion $(\boldsymbol{n}=\mathbf{2 4})$ & Pre-Erosion $(\boldsymbol{n}=\mathbf{6 2})$ \\
\hline Device Characteristics & & \\
\hline Device type $n(\%)$ & & \\
- VR PPM & $6(25 \%)$ & $4(6.5 \%)$ \\
- DR PPM & $14(58.3 \%)$ & $22(35.5 \%)$ \\
- CRTD & $1(4.2 \%)$ & $11(17.8 \%)$ \\
- CRTP & $3(12.5 \%)$ & $16(25.8 \%)$ \\
- VR ICD & $0(0 \%)$ & $2(3.2 \%)$ \\
- DR ICD & $0(0 \%)$ & $7(11.3 \%)$ \\
Number of leads & & \\
- Mean +/-SD & $2.39+/-0.8$ & $2.48+/-0.74$ \\
Lead dwell time (years) & & \\
- Mean +/-SD & $17.87+/-6.32$ & \\
Lead fixation $n(\%)$ & $1(4.3 \%)$ & $22(35.5 \%)$ \\
- Active & $10(41.7 \%)$ & $2(3.2 \%)$ \\
- Passive & $9(37.5 \%)$ & $31(50 \%)$ \\
- Both & $4(16.7 \%)$ & $7(11.3 \%)$ \\
- Unknown & \\
\hline Values are mean $+/-$ standard deviation $(\mathrm{SD})$ or $n(\%)$ & \\
\hline
\end{tabular}

The mean white cell count (WCC) was $7.4(+/-2.14)$, and the mean CRP was 28 . No vegetations were seen on echocardiography. All patients in the pre-erosion group were apyrexial with negative blood cultures and no evidence of systemic infection. One patient in the erosion group had a positive blood culture (Staphylococcus epidermidis). This patient was 86-years-old with a CFS score of 7 and a lead dwell time of 8 years, and initially opted for a conservative strategy but required a full system extraction during the same admission and died 11 months after the index revision. There were no positive pocket swab cultures.

The TYRX antibacterial envelope became available to us in August 2016. A total of 26 out of the $43(53.49 \%)$ procedures undertaken thereafter involved the use of a TYRX antibacterial envelope at the time of revision. Overall, 7 patients required further intervention (a repeat reburial or an extraction) after the utilization of a TYRX antibacterial envelope during their index reburial procedure.

The mean age in the erosion group was 85 years, with a CFS of 4.98 and a lead dwell time of 18 years. By comparison, the pre-erosion group had a mean age of 68 years, a CFS of 3.98, and a dwell time of 8 years.

The mean length of stay was longer in the erosion group at 3.2 days versus $<1$ day in the pre-erosion group. The mean duration of antibiotic therapy post-procedure for the whole cohort was 5.8 days.

There was no in-hospital mortality, and all patients were alive at 30 days postprocedure. Overall, $7(8 \%)$ patients had a repeat procedure (including 1 patient who underwent 3 revisions), and $13(15 \%)$ went on to undergo a CIED extraction during the follow-up period. See Table 2 .

Overall, 7 (8.1\%) deaths occurred within 12 months of revision. A total of $21(24.4 \%)$ patients died during the follow-up period. A higher proportion of patients presenting with erosion died within 12 months of revision ( $16.67 \%$ vs $4.84 \%$ ). Further, $25 \%$ with an eroded device and $11 \%$ with a pre-eroding device who underwent revision required an extraction during the follow-up period.

At 12 months post-procedure, more patients in the erosion group had died compared to the pre-erosion group ( $17 \%$ vs $5 \%, p=0.16)$. This trend continued over the mediumterm follow-up ( $42 \%$ vs $18 \%, p=0.04)$. At the last available follow-up, $13(15 \%)$ patients went on to a full system extraction: 6/24 (25\%) in the erosion group and 7/62 (11\%) in the pre-erosion group. Figures 1 and 2 show Kaplan-Meier curves for survival and further reintervention. 
Table 2. Outcomes.

\begin{tabular}{|c|c|c|}
\hline Outcomes & Erosion $(n=24)$ & Pre-Erosion $(n=62)$ \\
\hline Number of patients requiring re-intervention (after index reburial) $n(\%)$ & $4(16.67 \%)$ & $3(4.84 \%)$ \\
\hline Number of re-intervention procedures & 7 & 3 \\
\hline Extraction $n(\%)$ & $6(25 \%)$ & $7(11.29 \%)$ \\
\hline Death during follow-up period $n(\%)$ & $10(41.67 \%)$ & $11(17.74 \%)$ \\
\hline Death within 12 months of index reburial $n(\%)$ & $4(16.67 \%)$ & $3(4.84 \%)$ \\
\hline Mean time to death following index procedure (months) Mean $+/-$ SD & $22.6(+/-20.3)$ & $30.4(+/-18)$ \\
\hline
\end{tabular}

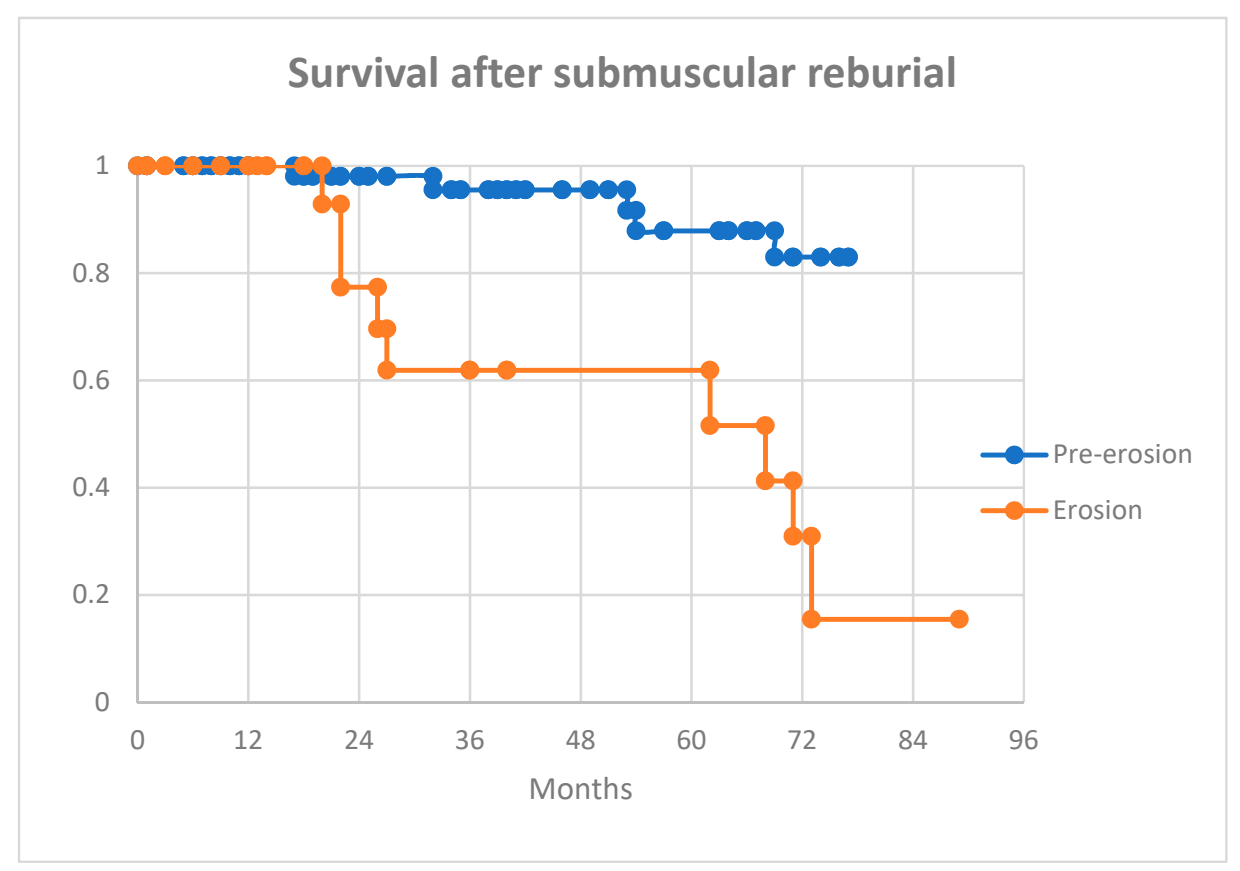

Figure 1. Kaplan-Meier curve of survival after submuscular reburial.

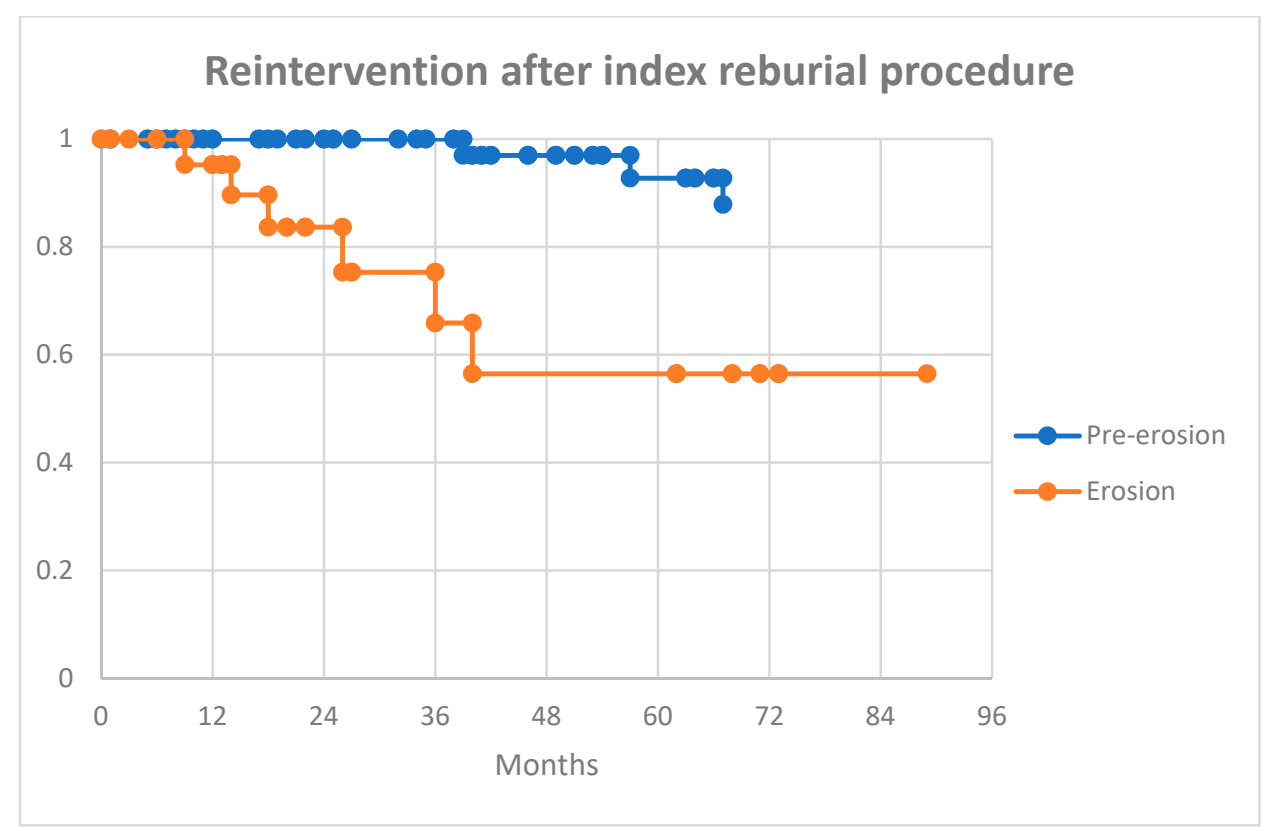

Figure 2. Kaplan-Meier curve showing the need for reintervention after the index reburial procedure. 


\section{Discussion}

When patients present with a CIED infection, the alternatives to TLE have to be explored at the time of consent, and-in order to facilitate meaningful dialogue - an idea of the expected outcomes for individual patients is important.

We believe that our paper enables clinicians to select patients who may be offered an alternative approach to lead extraction, especially where patient choice or perceived risk precludes exploring TLE as a primary strategy. The short-, medium-, and long-term outcome data we present from our cohort can guide informed consent.

In a recent paper, Santoro et al. reported that the efficacy and safety of transvenous lead extraction in octogenarian patients were comparable to those of a younger cohort [20]. This was a retrospective review with some important caveats: patient outcome data were not available, a frailty assessment of the elderly patients was not performed, and the median lead dwell time was 66 months (appreciably shorter than our 118 months).

Our paper reports outcomes of conservative management in two distinct cohorts of patients presenting with CIED issues-those with erosion and pre-erosion.

The overall $8 \%$ mortality rate at 12 months post-revision compares very favourably with a $44 \%$ mortality rate previously reported, despite comparable demography (a median age of 77.5 in our cohort versus a median age of 78). Even our erosion group fared better than reported, despite being significantly older [20]. The conservative management of CIED infections with positive blood cultures was associated with poor outcomes, despite chronic antibiotic suppression [21-23].

Another notable difference was the infrequency of chronic antibiotic suppression in our cohort, mainly guided by clinical presentation and infective markers. We only employed four-week intravenous antibiotics in two patients-one with bacteraemia and the second due to clinical concerns. We propose the main reason for these findings was the absence of systemic infection in all but one of our patients. Additionally, we had no positive culture results from the pocket swabs obtained, resulting in a reduced overall mean duration of antibiotic therapy.

Our cohort-particularly the erosion group-is not reflected in extraction registries, including ELECTRA, which reported a mean age of 65 years and a mean dwell time of 6.4 years on the leads [11]; therefore, the risk of $\sim 2 \%$ at extraction may be underestimated.

We observed that 55/86 (64\%) patients underwent a procedure in the 12 months prior to presentation. This is in keeping with data reporting an almost $10 \%$ complication rate post pacing intervention [24].

Over two-thirds (69\%) of our patients were alive with no further intervention required at 12 months.

\section{Limitations}

This was a retrospective observational study; however, even with all the shortcomings it entails, the longitudinal outcome data provide important insight into this cohort.

The cause of death was unavailable but may have clarified if it was related to general frailty and age or due to CIED infection itself.

\section{Conclusions}

As the prevalence of CIEDs increases, issues with CIEDs are likely to be encountered more frequently. Individually tailored strategies need to be deployed in a timely manner for good outcomes.

We believe our data provide important outcome information on an alternative strategy to TLE in carefully selected patients where the extraction risk is perceived to be unacceptable. The absence of systemic infection appears to predict better outcomes in this cohort, and two-thirds of patients remained complication-free at 12 months.

We believe that these data will guide informed consent and enable clinicians to manage patients appropriately. 


\begin{abstract}
Author Contributions: Conceptualization, J.L. and A.R.; methodology, J.L. and A.R.; formal analysis, J.L.; investigation, G.M., A.R. and J.L.; resources, A.R. and D.J.W.; data curation, G.M., A.R. and J.L.; writing—original draft preparation, J.L. and A.R.; writing—review and editing, J.L., D.G., D.J.W. and A.R.; visualization, J.L.; supervision, A.R.; project administration, A.R. All authors have read and agreed to the published version of the manuscript.
\end{abstract}

Funding: This research received no external funding.

Institutional Review Board Statement: This study was conducted in accordance with the Declaration of Helsinki and approved by the Institutional Review Board (or Ethics Committee) of the Liverpool Heart and Chest Hospital clinical audit and effectiveness group (protocol code 320 and date of approval 16 September 2020).

Informed Consent Statement: Patient consent was waived as the study involved retrospective review of medical records of all patients who had undergone submuscular reburial between 2013 and 2020. The results of the research will not affect clinical care of the patients since the management strategy had already been implemented.

Data Availability Statement: The data supporting the findings of this study are available within the article.

Conflicts of Interest: Professor Wright and Dr. Rao have received payment for consulting on an advisory board for Medtronic and for educational lectures provided to Medtronic and Boston Scientific. The remaining authors have declared no competing interests for this manuscript.

\title{
References
}

1. Kusumoto, F.M.; Schoenfeld, M.H.; Wilkoff, B.L.; Berul, C.I.; Birgersdotter-Green, U.M.; Carrillo, R.; Cha, Y.-M.; Clancy, J.; Deharo, J.-C.; Ellenbogen, K.A.; et al. 2017 HRS expert consensus statement on cardiovascular implantable electronic device lead management and extraction. Heart Rhythm 2017, 14, 503-551. [CrossRef]

2. Hindricks, G.; Camm, J.; Merkley, B.; Raatikainen, P.; Arnar, D.O. The EHRA White Book 2017. 2017. Available online: https: //www.escardio.org/static-file/Escardio/Subspecialty/EHRA/Publications/Documents/2017/ehra-white-book-2017.pdf (accessed on 24 March 2021).

3. Uslan, D.Z.; Tleyjeh, I.M.; Baddour, L.M.; Friedman, P.A.; Jenkins, S.M.; St Sauver, J.L.; Hayes, D.L. Temporal trends in permanent pacemaker implantation: A population-based study. Am. Heart J. 2008, 155, 896-903. [CrossRef] [PubMed]

4. Krahn, A.D.; Longtin, Y.; Philippon, F.; Birnie, D.H.; Manlucu, J.; Angaran, P.; Rinne, C.; Coutu, B.; Low, R.A.; Essebag, V.; et al. Prevention of Arrhythmia Device Infection Trial: The PADIT Trial. J. Am. Coll. Cardiol. 2018, 72, 3098-3109. [CrossRef] [PubMed]

5. Tarakji, K.G.; Mittal, S.; Kennergren, C.; Corey, R.; Poole, J.E.; Schloss, E.; Gallastegui, J.; Pickett, R.A.; Evonich, R.; Philippon, F.; et al. Antibacterial Envelope to Prevent Cardiac Implantable Device Infection. N. Engl. J. Med. 2019, 380, $1895-1905$. [CrossRef] [PubMed]

6. $\quad$ Clementy, N.; Carion, P.L.; de Leotoing, L.; Lamarsalle, L.; Wilquin-Bequet, F.; Brown, B.; Verhees, K.J.P.; Fernandes, J.; Deharo, J.-C. Infections and associated costs following cardiovascular implantable electronic device implantations: A nationwide cohort study. Europace 2018, 20, 1974-1980. [CrossRef] [PubMed]

7. Blomstrom-Lundqvist, C.; Traykov, V.; Erba, P.A.; Burri, H.; Nielsen, J.C.; Deharo, J.-C.; Epstein, L.M.; Saghy, L.; Snygg-Martin, U.; Starck, C.; et al. European Heart Rhythm Association (EHRA) international consensus document on how to prevent, diagnose, and treat cardiac implantable electronic device infections-endorsed by the Heart Rhythm Society (HRS), the Asia Pacific Heart Rhythm Society (APHRS). Europace 2020, 22, 515-549. [CrossRef]

8. Li, J.S.; Sexton, D.J.; Mick, N.; Nettles, R.; Fowler, V.G., Jr.; Ryan, T.; Bashore, T.; Corey, G.R. Proposed modifications to the Duke criteria for the diagnosis of infective endocarditis. Clin. Infect. Dis. 2000, 30, 633-638. [CrossRef] [PubMed]

9. Lebeaux, D.; Fernández-Hidalgo, N.; Chauhan, A.; Lee, S.; Ghigo, J.M.; Almirante, B.; Beloin, C. Management of infections related to totally implantable venous-access ports: Challenges and perspectives. Lancet Infect. Dis. 2014, 14, 146-159. [CrossRef]

10. Deharo, J.C.; Bongiorni, M.G.; Rozkovec, A.; Bracke, F.; Defaye, P.; Fernandez-Lozano, I. Pathways for training and accreditation for transvenous lead extraction: A European Heart Rhythm Association position paper. Europace 2012, 14, $124-134$.

11. Bongiorni, M.G.; Kennergren, C.; Butter, C.; Deharo, J.C.; Kutarski, A.; Rinaldi, C.; Romano, S.L.; Maggioni, A.P.; Andrala, M.; Auricchio, A.; et al. The European Lead Extraction ConTRolled (ELECTRa) study: A European Heart Rhythm Association (EHRA) Registry of Transvenous Lead Extraction Outcomes. Eur. Heart J. 2017, 38, 2995-3005. [CrossRef]

12. Agarwal, S.K.; Kamireddy, S.; Nemec, J.; Voigt, A.; Saba, S. Predictors of complications of endovascular chronic lead extractions from pacemakers and defibrillators: A single-operator experience. J. Cardiovasc. Electrophysiol. 2009, 20, 171-175. [CrossRef]

13. Alonso, C.; Marquie, C.; Defaye, P.; Clementy, N.; Mondoly, P.; Sadoul, N.; Boveda, S.; Hidden-Lucet, F.; Dompnier, A.; Da Costa, A.; et al. FRAGILE: FRench Attitude reGistry in case of ICD LEad replacement. Ep Eur. 2021, 23, 389-394. [CrossRef] [PubMed]

14. Church, S.; Rogers, E.; Rockwood, K.; Theou, O. A scoping review of the Clinical Frailty Scale. BMC Geriatr. 2020, 20, 393. [CrossRef] [PubMed] 
15. Todd, F.; Wong, C.; Hewitson, L.; Mohamed, A.; Doolub, J.; Gogola, L.; Skyrme-Jones, A.; Aziz, S.; Sammut, E.; Dastidar, A. Prognostic role of the Rockwood Clinical Frailty Scale in patients with acute heart failure:results from a single-centre retrospective cohort study. Eur. Heart J. Acute Cardiovasc. Care 2021, 10, zuab020-042. [CrossRef]

16. Rockwood, K.; Song, X.; MacKnight, C.; Bergman, H.; Hogan, D.B.; McDowell, I.; Mitnitski, A. A global clinical measure of fitness and frailty in elderly people. CMAJ 2005, 173, 489-495. [CrossRef] [PubMed]

17. General Medical Council. Available online: https://www.gmc-uk.org/-/media/documents/updated-decision-making-andconsent-guidance_pdf-84160128.pdf (accessed on 15 March 2021).

18. Lopez, J.A. Conservative management of infected pacemaker and implantable defibrillator sites with a closed antimicrobial irrigation system. Europace 2013, 15, 541-545. [CrossRef] [PubMed]

19. Poller, W.C.; Schwerg, M.; Melzer, C. Therapy of cardiac device pocket infections with vacuum-assisted wound closure-Longterm follow-up. Pacing Clin. Electrophysiol. 2012, 35, 1217-1221. [CrossRef]

20. Giannotti Santoro, M.; Segreti, L.; Zucchelli, G.; Barletta, V.; Fiorentini, F.; Di Cori, A.; De Lucia, R.; Bongiorni, M.G. Transvenous lead extraction: Efficacy and safety of the procedure in octogenarian patients. Pacing Clin. Electrophysiol. 2020, 43, 382-387. [CrossRef]

21. Tan, E.M.; DeSimone, D.C.; Sohail, M.R.; Baddour, L.M.; Wilson, W.R.; Steckelberg, J.M.; Virk, A. Outcomes in Patients with Cardiovascular Implantable Electronic Device Infection Managed with Chronic Antibiotic Suppression. Clin. Infect. Dis. 2017, 64, 1516-1521. [CrossRef]

22. Utili, R.; Durante-Mangoni, E.; Tripodi, M.F. Infection of intravascular prostheses: How to treat other than surgery. Int. J. Antimicrob. Agents 2007, 30, 42-50. [CrossRef]

23. Rundström, H.; Kennergren, C.; Andersson, R.; Alestig, K.; Hogevik, H. Pacemaker endocarditis during 18 years in Göteborg. Scand. J. Infect. Dis. 2004, 36, 674-679. [CrossRef] [PubMed]

24. Kirkfeldt, R.E.; Johansen, J.B.; Nohr, E.A.; Jørgensen, O.D.; Nielsen, J.C. Complications after cardiac implantable electronic device implantations: An analysis of a complete, nationwide cohort in Denmark. Eur. Heart J. 2014, 35, 1186-1194. [CrossRef] [PubMed] 